Pacific Journal of Mathematics

WEIGHTED SIDON SETS 


\title{
WEIGHTED SIDON SETS
}

\section{J. W. SANDERS}

\begin{abstract}
A weighted generalisation of Sidon sets, $W$-Sidon sets, is introduced and studied for compact abelian groups. Firstly $W$-Sidon sets are characterised analogously to Sidon sets and variations of these characterisations shown to lead back to Sidon sets. For the circle group $W$-Sidon sets are constructed which are not $\Lambda(1)$ and hence not Sidon. The algebra of all $W$ 's making a set $W$-Sidon is investigated and Sidon and $p$-Sidon sets cast in terms of it. Finally analytic properties of $W$-Sidon sets are pursued and a necessary condition on the growth of $W^{2}$ obtained.
\end{abstract}

Throughout this paper $G$ denotes a compact abelian Hausdorff topological group and $X$ denotes its (discrete) dual group. Both are written multiplicatively with identities $e$ and 1 respectively.

We write $\left(L^{p}(G),\|\cdot\|_{p}\right)$ for the Lebesgue space derived from the normalised Haar measure on $G$ and $\left(C(G),\|\cdot\|_{\infty}\right)$ for the space of (complex-valued) functions continuous on $G$ with the supremum norm. However for $\Delta \subseteq X$ and counting measure on $\Delta$ we denote the Lebesgue spaces $\left(l^{p}(\Delta),\|\cdot\|_{p}\right)$ and use $c_{0}(\Delta)$ for the subset of $l^{\infty}(\Delta)$ of functions tending to zero at infinity.

If $A$ and $B$ are sets we write $B^{A}$ for the set of all functions from $A$ to $B$; if $f \in B^{A}$ and $C \subseteq A$ ( $\subset$ is reserved for strict inclusion) we write $f \mid C$ for the restriction of $f$ to $C ; \xi_{A}$ is the characteristic function of $A$; $\mathfrak{F}(A)$ denotes the set of all finite subsets of $A$; $\mathfrak{P}(A)$ denotes the power set of $A ; \nu(A)$ is the cardinality of $A$; and we write $\square$ for the empty set.

The sets of complex numbers, real numbers, integers and natural numbers will be written $\mathfrak{F}, \mathfrak{R}, 3$, and $\mathfrak{N}$ respectively and we write $\mathfrak{T}$ for the topological group of unimodular complex numbers. If $c \in \mathfrak{F}$, c denotes the constant function with value $c$, whose domain will be clear from the context.

For $\Delta \subseteq X, \phi \in \mathfrak{S}^{\Delta}$ and $A \subseteq \complement^{\Delta}$ we write $\phi A$ for $\{\phi \psi: \psi \in A\}$.

We denote the Fourier transform of $f \in L^{1}(G)$ by $\hat{f}$. If $E$ is a Banach space we write $E^{\prime}$ for its dual. Let $A(G)=\left\{f \in C(G): \hat{f} \in l^{1}(X)\right\}$ be normed by $\|f\|_{A}=\|\hat{f}\|_{1}$ and set the space of pseudomeasures on $G$, $\left(P M(G),\|\cdot\|_{P M}\right)$, equal to $A(G)^{\prime}$ so that it contains $(M(G),\|\cdot\|)$, the space of measures on $G$. For $\pi \in P M(G)$ we write $\hat{\pi}$ for its Fourier transform and $s p \pi$ for its spectrum, i.e. $\{\chi \in X: \hat{\pi}(\chi) \neq 0\}$. If $E \subseteq$ $P M(G)$ and $\Delta \subseteq X$ we let $E_{\Delta}=\{\pi \in E: s p \pi \subseteq \Delta\}$ and call its members $\Delta$-spectral pseudomeasures. We also write $E^{\wedge}$ for $\{\hat{\pi}: \pi \in E\}$. 
The set of trigonometric polynomials on $G$ will be denoted $T(G)$. A subset $\Delta$ of $X$ is called

(i) a Sidon set iff $\sup \left\{\sum_{x \in \Delta}|\hat{t}(\chi)|: t \in T_{\Delta}(G)\right.$ and $\left.\|t\|_{\infty} \leqq 1\right\}<\infty$, and

(ii) a $\Lambda(p)$ set, for $0<p<\infty$ (written $\Delta \in \Lambda(p)$ ) iff for some $r$ with $0<r<p, L_{\Delta}^{p}(G)=L_{\Delta}^{r}(G)$. The reader is referred to [2] for an exposition of Sidon and $\Lambda(p)$ sets.

\section{W-Sidon sets.}

Definitions 1.0. If $\Delta \subseteq X$ and $W \in \mathbb{\Im}^{\Delta}$ we let

$$
\|W\|_{\Delta}=\sup \left\{\sum_{\chi \in A}|W(\chi) \hat{t}(\chi)|: t \in T_{\Delta}(G) \text { and }\|t\|_{\infty} \leqq 1\right\}
$$

and say $\Delta$ is $W$-Sidon iff this is finite. Set

$$
\mathfrak{W}(\Delta)=\left\{W \in \mathbb{C}^{\Delta}:\|W\|_{\Delta}<\infty\right\} \text {. }
$$

Evidently $\|W\|_{\Delta}$ equals the least constant for which, whenever $t \in T_{\Delta}(G), \sum_{\chi \in \Delta}|W(\chi) \hat{t}(\chi)| \leqq k\|t\|_{\infty}$.

The letter $W$ is used to suggest a weight function and $W$-Sidon sets should not be confused with $p$-Sidon sets ([4]) or $V$-Sidon sets ([13]).

1.1. Taking $\chi \in \Delta$ as $t$ above we see $\|W\|_{\infty} \leqq\|W\|_{\Delta}$. So $\Delta$ is. Sidon iff $\mathfrak{W}(\Delta)=l^{\infty}(\Delta)$ and the Sidon constant of $\Delta$ equals $\|1\|_{\Delta}$.

\subsection{For any $\Delta \leqq X, l^{2}(\Delta) \subseteq \mathfrak{W}(\Delta)$.}

For if $t \in T(G)$ the Cauchy-Schwarz inequality followed by Parseval's identity shows

$$
\sum_{\chi \in \Delta}|W(\chi) \hat{t}(\chi)| \leqq\|W\|_{2}\|\hat{t}\|_{2}=\|W\|_{2}\|t\|_{2} \leqq\|W\|_{2}\|t\|_{\infty} .
$$

Thus $\|W\|_{4} \leqq\|W\|_{2}$.

In the $W$-Sidon theory to follow, sets $\Delta$ for which $W \in l^{2}(\Delta)$ behave very like finite sets in the Sidon theory. We refer to them as trivial $W$-Sidon sets.

Examples of $\Delta$ and $W$ for which $W \notin l^{2}(\Delta)$ yet $\Delta$ is $W$-Sidon and not Sidon are given in 2.3, and some infinite $\Delta$ 's which are $W$-Sidon only for $W \in l^{2}(\Delta)$ in 3.4 .

1.3. In 1.0 we have not referred directly to the group $X$. The following result excuses this. Let $X_{1}$ and $X_{2}$ be discrete abelian groups with $\Delta \subseteq X_{1}$ and $X_{1}$ a subgroup of $X_{2}$.

THEOREM. For $W \in \mathbb{C}^{\Delta}, \Delta$ is $W$-Sidon as a subset of $X_{1}$ iff it 
is W-Sidon as a subset of $X_{2}$.

Proof. Suppose that $G_{i}$ is the dual of $X_{i}$ for $i \in\{1,2\}$ and define an equivalence relation $\alpha$ on $G_{1}$ by $(x, y) \in \alpha$ iff $\chi(x)=\chi(y)$ for all $\chi \in X_{1}$. Writing $A$ for $\left\{x \in G_{1}: \chi(x)=1\right.$ for all $\left.\chi \in X_{1}\right\}$, the kernel of $\alpha, A$ is a closed subgroup of $G_{1}$ and $G_{1} / A$ is isomorphic to $G_{2}$ by [10], 2.1.

For $t \in T_{\Delta}\left(G_{2}\right)$ define $t^{*} \in T_{\Delta}\left(G_{1} / A\right)$ by

$$
t^{*}(\alpha(x))=\sum_{\chi \in \Delta} \hat{t}(\chi) \chi(x) .
$$

By definition of $\alpha$, the map $\beta: T_{4}\left(G_{2}\right) \rightarrow T_{4}\left(G_{1} / A\right)$ given by $\beta(t)=t^{*}$ is well defined. It is easily seen to be a vector space isomorphism, $\|\cdot\|_{\infty}$-isometric and to satisfy

$$
(\beta(t))^{\wedge}(\chi)=\hat{t}(\chi) \text { for all } t \in T_{\Delta}\left(G_{2}\right) \text { and } \chi \in \Delta .
$$

Consequently

$$
\begin{aligned}
& \sup \left\{\sum_{\chi \in \Delta}|W(\chi) \hat{t}(\chi)|: t \in T_{\Delta}\left(G_{2}\right) \text { with }\|t\|_{\infty} \leqq 1\right\} \\
= & \sup \left\{\sum_{\chi \in \Delta}|W(\chi) \hat{u}(\chi)|: u \in T_{\Delta}\left(G_{1} / A\right) \text { with }\|u\|_{\infty} \leqq 1\right\}
\end{aligned}
$$

and the conclusion follows.

1.4. To see how $W$-Sidon sets are affected by group operations on $X$ we extend 1.3 as follows. If $\phi$ is a function from one discrete abelian group $X_{1}$ to another, $X_{2}$, (with duals $G_{i}$ ) it induces a map $\phi^{*}$ from $T\left(G_{1}\right)$ to $T\left(G_{2}\right)$ by

$$
\sum_{\chi \in X_{1}} \hat{t}(\chi) \chi \longmapsto \sum_{\chi \in X_{1}} \hat{t}(\chi) \phi(\chi)
$$

When $\phi^{*}$ is $\|\cdot\|_{\infty}$-isometric, $\phi$ is injective so given $\Delta \cong X$ and $W \in \mathbb{C}^{\Delta}$ there is a map $W_{\phi} \in \mathfrak{C}^{\phi}$ defined by

$$
W_{\phi}(\phi(\chi))=W(\chi) \text { for all } \chi \in \Delta .
$$

THeOREM. If $\phi^{*}$ is $\|\cdot\|_{\infty}$-isometric, $\Delta$ is W-Sidon iff $\phi(\Delta)$ is $W_{\phi}$-Sidon.

Proof. Now $\phi^{*}$ maps $T_{\Delta}\left(G_{1}\right)$ onto $T_{\phi(\Delta)}\left(G_{2}\right)$ and whenever $t \in T_{\Delta}\left(G_{1}\right)$ and $\chi \in \Delta$,

$$
W(\chi) \hat{t}(\chi)=W_{\phi}(\phi(\chi))\left(\phi^{*} t\right)^{\wedge}(\phi(\chi)) .
$$

Consequently, using 1.3 to move from the group $\phi\left(X_{1}\right)$ to $X_{2}$, 


$$
\begin{aligned}
\|W\|_{\Lambda} & =\sup \left\{\sum_{\chi \in \Delta}|W(\chi) \hat{t}(\chi)|: t \in T_{\Delta}\left(G_{1}\right) \text { and }\|t\|_{\infty} \leqq 1\right\} \\
& =\sup \left\{\sum_{\xi \in \phi(\Delta)}\left|W_{\phi}(\xi) \hat{u}(\xi)\right|: u \in T_{\phi(\Delta)}\left(G_{2}\right) \text { and }\|u\|_{\infty} \leqq 1\right\} \\
& =\left\|W_{\phi}\right\|_{\phi(\Delta)} .
\end{aligned}
$$

1.5. (i) For example take as $\phi$ the map $\tau_{\chi_{0}}: X \rightarrow X$ (for $\chi_{0} \in X$ ) given by $\tau_{\chi_{0}}(\chi)=\chi_{0} \chi$. If $t \in T(G)$,

$$
\left\|\tau_{\chi_{0}}^{*}(t)\right\|_{\infty}=\left\|\sum_{\chi \in X} \hat{t}(\chi) \chi_{0} \chi\right\|_{\infty}=\left\|\sum_{\chi \in X} \hat{t}(\chi) \chi\right\|_{\infty}
$$

whence $\tau_{\chi_{0}}^{*}$ is $\|\cdot\|_{\infty}$-isometric. For any $\Delta \subseteq X, \chi_{0} \in X$ and $W \in \mathbb{C}^{4}$, provided we define $W_{0} \in \mathfrak{C}^{\chi_{0} \Delta}$ by $W_{0}\left(\chi_{0} \chi\right)=W(\chi)$ for all $\chi \in \Delta, 1.4$ guarantees

$$
\mathfrak{W}\left(\chi_{0} \Delta\right)=\left\{W_{0}: W \in \mathfrak{W}(\Delta)\right\}
$$

(ii) Similarly if we define $\rho: X \rightarrow X$ by $\rho(\chi)=\chi^{-1}$ then provided we set $W_{\rho} \in \mathfrak{( ⿻ 上 丨 - 1 ~}^{\Lambda_{-1}}$ to be $W_{\rho}\left(\chi^{-1}\right)=W(\chi), 1.4$ shows

$$
\mathfrak{B}\left(\Delta^{-1}\right)=\left\{W_{\rho}: W \in W(\Delta)\right\} \text {. }
$$

(iii) Note that for $W \in \mathcal{S}^{\Delta \cup \chi_{0} \Delta}, 1.5$ (i) does not claim $\Delta$ is $W$-Sidon iff $\chi_{0} \Delta$ is $W$-Sidon (and similarly for 1.5(ii)).

If $\Delta$ is an infinite proper subgroup of $X$ (it can be chosen for 3 say) and $\chi_{0} \in X \backslash \Delta$ then clearly $\chi_{0} \Delta \cap \Delta=\square$. So we may choose $W \in \mathfrak{C}^{\Delta \cup \chi_{0} \Delta}$ such that $W \mid \Delta \in l^{2}(\Delta)$ yet $W \mid \chi_{0} \Delta \in l^{\infty}\left(\chi_{0} \Delta\right) \backslash l^{2}\left(\chi_{0} \Delta\right)$. A premature glance at 3.3 now shows, together with 1.5(i), that $\mathfrak{W}(\Delta)=l^{2}(\Delta)$ and $\mathfrak{W}\left(\chi_{0} \Delta\right)=$ $l^{2}\left(\chi_{0} \Delta\right)$. Thus $\Delta$ is $W$-Sidon yet $\chi_{0} \Delta$ is not $W$-Sidon (taking restrictions for granted).

1.6. Suppose $E$ is a Banach space contained in $P M(G)$, with norm $\|\cdot\|_{E}$ stronger than $\|\cdot\|_{P M}$. For $\Delta \subseteq X$ define $\delta: E \rightarrow E^{\wedge} \mid \Delta$ by $\delta(\pi)=\hat{\pi} \mid \Delta$. Since $\delta$ is a vector space morphism, ker $\delta$ is a subspace of $E$. This subspace is closed since if $\pi \in E$ and $\left\{\pi_{n}: n \in \mathfrak{R}\right\} \subseteq \operatorname{ker} \delta$ with $\left\|\pi-\pi_{n}\right\|_{E} \rightarrow 0$ then $\left\|\hat{\pi}-\hat{\pi}_{n}\right\|_{\infty} \rightarrow 0$ hence $\hat{\pi} \mid \Delta=\mathbf{0}$.

Thus $E / \operatorname{ker} \delta$ is a Banach space under the quotient norm. Equivalently, $E^{\wedge} \mid \Delta$ is a Banach space with norm

$$
\|\phi\|_{\delta}=\inf \left\{\|\pi\|_{E}: \pi \in E \text { and } \hat{\pi} \mid \Delta=\phi\right\} .
$$

Evidently for all $\pi \in E$,

$$
\|\hat{\pi}\|_{\infty} \leqq\|\hat{\pi} \mid \Delta\|_{\delta} \leqq\|\pi\|_{E}
$$

(See also 3.7.)

If $E$ is a Banach subalgebra of $P M(G)$ (not necessarily with identity) then so too is $E^{\wedge} \mid \Delta$. 
When considering $E^{\prime}$ rather than $E$ we write $\delta^{\prime}$ in place of $\delta$.

1.7. Our dependence on $\Delta$-spectral functions makes the following result useful. Refer to [7], Chapter 1, (2.10) for the definition of a homogeneous Banach space on $G$, replacing $\mathfrak{I}$ there by $G$.

Suppose $E$ is a homogeneous Banach space on $G$ and $E^{\prime}$ is the dual of $E$ under a pairing $\langle f, \psi\rangle$ (for $f \in E$ and $\psi \in E^{\prime}$ ). If $\psi \in E^{\prime}$ and $\chi \in X \cap E$ then the Fourier coefficient is defined to be

$$
\hat{\psi}(\chi)=\langle\overline{\chi, \psi}\rangle
$$

and satisfies $|\hat{\psi}(\chi)| \leqq\|\psi\|_{E^{\prime}}\|\chi\|_{E}$.

THeOREM. Let $\Delta \subseteq X$, let $E$ be a homogeneous Banach space on $G$ containing $\triangle$ and suppose that, restricted to $\Delta,\|\cdot\|_{E}$ is weaker than $\|\cdot\|_{A}$. Then there is a canonical isomorphism from $\left(E_{A}\right)^{\prime}$ to $\left(E^{\prime}\right)^{\wedge} \mid \Delta$ (the latter being normed by $\left.\|\cdot\|_{\delta^{\prime}}\right)$ whose norm is less than or equal to one.

Proof. Since

$$
\|\hat{f}\|_{\infty} \leqq\|f\|_{1} \leqq\|f\|_{E}, \text { for all } f \in E,
$$

$E_{\Delta}$ is a closed subspace of $E$. So the canonical map

$$
J:\left(E_{4}\right)^{\prime} \longrightarrow E^{\prime} /\left(E_{4}\right)^{0}
$$

is an isomorphism of norm less than or equal to 1 , where $\left(E_{4}\right)^{0}$, the annihilator of $E_{\Delta}$, is $\left\{\psi^{\prime} \in E^{\prime}: \psi(f)=0\right.$ for all $\left.f \in E_{\Delta}\right\}$ (see [8], p. 93).

Now $|\hat{\psi}(\chi)| \leqq\|\psi\|_{E^{\prime}}$ whenever $\psi \in E^{\prime}$ and $\chi \in \Delta$ thus by 1.6 it remains to show that $\left(E_{\Delta}\right)^{0}=\operatorname{ker} \delta^{\prime}$. If $\psi \in\left(E_{\Delta}\right)^{0}$ then $\psi(\chi)=0$ for all $\chi \in \Delta$ hence $\langle\chi, \psi\rangle=0$ so that $\hat{\psi} \mid \Delta=0$ whence $\psi \in \operatorname{ker} \delta^{\prime}$. Conversely if $\hat{\psi}(\chi)=0$ for all $\chi \in \Delta$ then $\psi(t)=0$ for all $t \in \operatorname{span}(\Delta)$. But span ( $\Delta)$ is dense in $E_{\Delta}$ (by the method of [7], Chapter 1, (2.12)) hence $\psi(f)=0$ whenever $f \in E_{\Delta}$, whence $\psi \in\left(E_{\Delta}\right)^{\circ}$.

Consequently $\left(E_{\Delta}\right)^{\prime}$ is isomorphic to $\left(E^{\prime}\right)^{\wedge} \mid \Delta$ under $J$ followed by the Fourier transform lifted to $E^{\prime} / \operatorname{ker} \delta^{\prime}$.

CoROLlary 1.8. Let $\Delta \leqq X$. Then

(i) if $1 \leqq p<\infty$, there is a canonical isomorphism from $L_{\Delta}^{p}(G)^{\prime}$ to $L^{p^{\prime}}(G)^{\wedge} \mid \Delta$ whose norm is dominated by 1 ,

(ii) there is a canonical isomorphism from $C_{\Delta}(G)^{\prime}$ to $M(G)^{\wedge} \mid \Delta$ whose norm is dominated by 1 , and

(iii) if $1 \leqq p<\infty$, there is a canonical isomorphism from $\left(L^{p}(G)^{\wedge} \mid \Delta\right)^{\prime}$ to $L_{\Delta}^{p^{\prime}}(G)$.

Proof. (i) and (ii) follow immediately from 1.7. 
If $1<p<\infty, L_{d}^{p^{\prime}}(G)$, being a closed subspace of the reflexive space $L^{p^{\prime}}(G)$, is also reflexive. So by (i) the dual of $L^{p}(G)^{\wedge} \mid \Delta$ is canonically isomorphic to $L_{\Delta}^{p^{\prime}}(G)^{\prime \prime}$, i.e. to $L_{\Delta}^{p^{\prime}}(G)$.

For $p=1$ we are forced to resort to the method of 1.7. Any $\psi \in\left(L^{1}(G)^{\wedge} \mid \Delta\right)^{\prime}$ lifts to a continuous linear map $\Psi: L^{1}(G) \rightarrow \mathbb{C}$ which is constant on cosets of $\operatorname{ker} \delta^{\prime}$ and which may be identified with an element of $L^{\infty}(G)$, giving $\|\Psi\|_{\infty} \leqq\|\psi\|$. Consequently if $\chi \in X \backslash 4$,

$$
\hat{\Psi}(\chi)=\int_{G} \psi \bar{\chi}=\int_{G} \psi \cdot 0=0
$$

so that $\Psi \in L_{\Delta}^{\infty}(G)$. This yields a map from $\left(L^{1}(G)^{\wedge} \mid \Delta\right)^{\prime}$ to $L_{\Delta}^{\infty}(G)$ and the method of 1.7 completes the proof.

REMARKS 1.9. (i) Obviously $A_{\Delta}(G)^{\prime}$ is isometrically isomorphic to $P M(G)^{\wedge} \mid \Delta$ as is $L_{\Delta}^{2}(G)^{\prime}$ to $L^{2}(G)^{\wedge} \mid \Delta$.

(ii) In (i) and (iii) above it suffices to take $\Delta=X$ to see the falsity for $p=\infty$. However $L_{\Delta}^{1}(G)$ can still be embedded canonically in $\left(L^{\infty}(G)^{\wedge} \mid \Delta\right)^{\prime}$, as can $C_{\Delta}(G)$ in $\left(M(G)^{\wedge} \mid \Delta\right)^{\prime}$.

THEOREM 1.10. Let $\Delta \subseteq X$ and $W \in \mathfrak{\Im}^{\Delta}$. With the understanding that the constants in (ii), (iii), (iv) and (v) are the least possible, the following are equivalent:

( i ) $\Delta$ is W-Sidon with $\kappa=\|W\|_{\Delta}$,

(ii ) $f \in L_{\Delta}^{\infty}(G)$ implies $\sum_{\chi \in \Delta}|W(\chi) \hat{f}(\chi)| \leqq \kappa\|f\|_{\infty}$,

(iii ) $f \in C_{\Delta}(G)$ implies $\sum_{\chi \in \Delta}|W(\chi) \hat{f}(\chi)| \leqq \kappa\|f\|_{\infty}$,

(iv) for all $\phi \in l^{\infty}(\Delta)$ there exists $\mu \in M(G)$ with $\hat{\mu} \mid \Delta=W \phi$ and $\|u\| \leqq \kappa\|\phi\|_{\infty}$,

( v ) for all $\phi \in c_{0}(\Delta)$ there exists $f \in L^{1}(G)$ with $\hat{f} \mid \Delta=W_{\phi}$ and $\|f\|_{1} \leqq \kappa\|\phi\|_{\infty}$

(vi) $W L_{\Delta}^{\infty}(G)^{\wedge} \mid \Delta \subseteq l^{1}(\Delta)$ (see section 0 for product notation),

(vii) $W C_{\Delta}(G)^{\wedge} \mid \Delta \subseteq l^{1}(\Delta)$,

(viii) $W l^{\infty}(\Delta) \cong M(G)^{\wedge} \mid \Delta$, and

( ix ) $W c_{0}(\Delta) \subseteq L^{1}(G)^{\wedge} \mid \Delta$.

Proof. (i) $\Rightarrow$ (ii) follows by a straightforward modification of (a) $\Rightarrow$ (b) in [10], 5.7.4.

(ii) $\Rightarrow$ (iii) is obvious because $C_{\Delta}(G) \subseteq L_{\Delta}^{\infty}(G)$.

(iii) $\Rightarrow$ (iv). By hypothesis the $\operatorname{map} f \mapsto W \hat{f} \mid \Delta$ from $C_{\Delta}(G)$ to $l^{1}(\Delta)$ is linear and bounded by $\kappa$. Let $K: l^{\infty}(\Delta) \rightarrow M(G)^{\wedge} \mid \Delta$ denote the canonical isomorphism of 1.8(ii) composed with the adjoint of this map-evidently $\|K\| \leqq \kappa$. For $\chi \in \Delta$,

$$
K \phi(\chi)=\sum_{\xi \in X} \phi(\xi)(W(\chi) \hat{\chi})(\xi)=W(\chi) \phi(\chi),
$$

so given $\phi \in l^{\infty}(\Delta)$, there is $\mu \in M(G)$-namely $\mu \in \delta^{-1}(K \phi)$-with $\hat{\mu} \mid \Delta=W \phi$ 
and $\|\mu\| \leqq \kappa\|\phi\|_{\infty}$.

(iv) $\Rightarrow$ (v) follows by an easy alteration of (d) $\Rightarrow(\mathrm{e})$ in [2], 15.1.4.

(v) $\Rightarrow$ (i). By hypothesis the map $\phi \mapsto W \phi$ from $c_{0}(\Delta)$ to $L^{1}(G)^{\wedge} \mid \Delta$ is linear and bounded by $\kappa$. Let $K: L_{\Delta}^{\infty}(G) \rightarrow l^{1}(\Delta)$ denote the composition of its adjoint with the canonical isomorphism of 1.8(iii). Then $K$ is linear and bounded by $\kappa$. If $\chi \in \Delta$ and $f \in L_{\Delta}^{\infty}(G)$ then

$$
(K f)(\chi)=\int_{G} W(\chi) f \bar{\chi}=W(\chi) \hat{f}(\chi)
$$

hence $K f=W \hat{f} \mid \Delta$, so (i) holds.

(ii) $\Rightarrow$ (vi), (iii) $\Rightarrow$ (vii), (iv) $\Rightarrow$ (viii) and (v) $\Rightarrow$ (ix) are obvious. Since the converses fall into similar pairs we show only one of each.

(vii) $\Rightarrow$ (iii). In the following lemma take $A$ to be $l^{1}(\Delta)$ with $\alpha$ the canonical injection, $B$ to be $C_{\Delta}(G)$ with $\beta f=W \hat{f} \mid \Delta$ and $C$ to be (5) with the product topology. Now (vii) ensures $\beta(B) \subseteq \alpha(A) \subseteq C$ so by 1.11 to follow, there is a constant $\kappa$ such that for all $f \in C_{\Delta}(G)$, there is $\phi \in l^{1}(\Delta)$ with $W \widehat{f} \mid \Delta=\phi$ and $\|\phi\|_{1} \leqq \kappa\|f\|_{\infty}$. That is, (iii) holds.

(ix) $\Rightarrow(\mathrm{v})$. In the following lemma take $A$ to be $L^{1}(G)$ with $\alpha(f)=\hat{f} \mid \Delta, B$ to be $c_{0}(\Delta)$ with $\beta(\phi)=W \phi$ and $C$ to be ${ }^{4}$ with the product topology. Now (ix) assures us that the hypotheses of 1.11 hold and hence (v) results.

1.11. I am indebted to Professor R. E. Edwards for the following statement:

Lemma. If $A$ and $B$ are Banach spaces, $C$ a Hausdorff topological vector space, $\alpha: A \rightarrow C$ and $\beta: B \rightarrow C$ continuous linear maps and if $\beta(B) \subseteq \alpha(A)$ then there is a constant $\kappa$ such that for all $b \in B$ there exists $a \in A$ with $\alpha(a)=\beta(b)$ and $\|a\|_{A} \leqq \kappa\|b\|_{B}$.

Proof. Let $\bar{A}=A / \operatorname{ker} \alpha$ and endow it with the quotient topology in which $\|\bar{a}\|=\inf \{\|c\|: c \in \bar{a}\}$ for each $\bar{a} \in \bar{A}$. Since $C$ is Hausdorff, $\{0\}$ is closed in $C$ and since $\alpha$ is continuous $\overline{0}=\alpha^{-1}(\{0\})$ is closed in $A$. Thus $\bar{A}$ is again a Banach space and $\alpha$ induces a continuous injection $\bar{\alpha}: \bar{A} \rightarrow C$ defined by $\bar{\alpha}(\bar{a})=\alpha(a)$, for $\bar{a} \in \bar{A}$.

Define $\gamma: B \rightarrow \bar{A}$ by $\gamma(b)=\bar{\alpha}^{-1} \circ \beta(b)$, for $b \in B$. By hypothesis $\gamma$ is well defined-it clearly suffices to show it is bounded. Evidently $\gamma$ is linear, so it remains to show it has a closed graph. If $b_{n} \rightarrow 0$ in $B$ and $\gamma\left(b_{n}\right) \rightarrow \bar{a}$ in $\bar{A}$ then $\beta\left(b_{n}\right) \rightarrow \beta(0)=0$ in $C$. Thus, since $\bar{\alpha}$ is also continuous and linear,

$$
\bar{\alpha}\left(\lim _{n} \gamma\left(b_{n}\right)\right)=\bar{\alpha}\left(\lim _{n} \bar{\alpha}^{-1} \circ \beta\left(b_{n}\right)\right)=\lim _{n} \beta\left(b_{n}\right)=\bar{\alpha}(\bar{\alpha})
$$

and so 


$$
0=\lim _{n} \beta\left(b_{n}\right)=\bar{\alpha}(\bar{a}) .
$$

Finally by injectivity of $\bar{\alpha}, \bar{a}=0$.

1.12. We shall also use this lemma in another direction.

THEOREm. Let $A$ and $B$ be Banach spaces, let $\triangle$ be a set and suppose $\mathbb{5}^{\Delta}$ has the product topology. Let $\alpha: A \rightarrow \mathfrak{\Im}^{\Delta}$ and $\beta: B \rightarrow \mathbb{C}^{\Delta}$ be continuous and linear with

(i) there is $\lambda>0$ such that for all $a \in A$ and all $\chi \in \Delta$,

$$
|\alpha(a)(\chi)| \leqq \lambda\|a\|_{A} \text {, and }
$$

(ii) there exist $\left\{b_{\chi}: \chi \in \Delta\right\} \subseteq B$ with

$$
\beta\left(b_{\chi}\right)(\xi)=\left\{\begin{array}{ll}
1 & \text { if } \xi=\chi \\
0 & \text { otherwise }
\end{array} \text {, and } \sup \left\{\left\|b_{\chi}\right\|_{B}: \chi \in \Delta\right\}<\infty .\right.
$$

Suppose finally that $\psi \in \mathbb{E}^{\Delta}$ with $\psi \beta(B) \subseteq \alpha(A)$. Then $\psi \in l^{\infty}(\Delta)$.

Proof. Applying 1.11 there is a constant $\kappa$ such that for all $b \in B$, there exists $a \in A$ with $\alpha(a)=\psi \beta(b)$ and $\|a\|_{A} \leqq \kappa\|b\|_{B}$. If we write $a_{\chi}$ for an element of $A$ corresponding to $b_{\chi}$ by this process we have

$$
|\psi(\chi)|=\left|\psi(\chi) \beta\left(b_{\chi}\right)(\chi)\right|=\left|\alpha\left(a_{\chi}\right)(\chi)\right| \leqq \lambda\left\|a_{\chi}\right\|_{A} \leqq \kappa \lambda\left\|b_{\chi}\right\|_{B} .
$$

Consequently $\|\psi\|_{\infty}<\infty$ as required.

1.13. The next result is helpful when showing a set is $W$-Sidon.

THEOREM. If $\Delta \subseteq X$ and $W \in \mathbb{C}^{\Delta}$ the following are equivalent:

(i) $\Delta$ is W-Sidon,

(ii) $f \in C_{\Delta}(G)$ with $\hat{f} \in \Re^{x}$ implies $\sum \chi \in \Delta|W(\chi) \hat{f}(\chi)|<\infty$, and

(iii) whenever $\phi \in l^{\infty}(\Delta) \cap \Re^{x}$ there is $\mu \in M(G)$ with $\hat{\mu} \mid \Delta=W \phi$.

Proof. (i) $\Rightarrow$ (ii) and (ii) $\Rightarrow$ (iii) follow from 1.10 .

(iii) $\Rightarrow$ (i). If $\phi \in l^{\infty}(\Delta)$ we may write $\phi=\phi_{1}+i \phi_{2}$ where, by (iii), there is $\mu_{j} \in M(G)$ with $\hat{\mu}_{j} \mid \Delta=W \phi_{j}$ for $j \in\{1,2\}$. Thus taking $\mu=\mu_{1}+i \mu_{2}$ gives $\mu \in M(G)$ and $\hat{\mu} \mid \Delta=W \phi$, so (i) results by 1.10 .

1.14. One important respect in which 1.10 differs from the analogous result for Sidon sets is that we only claim inclusions like 1.10 (viii) rather than $W l^{\infty}(\Delta)=M(G)^{\wedge} \mid \Delta$. The reasons for this are embodied in:

Theorem. Suppose $\Delta \subseteq X$ and $W \in \mathfrak{C}^{\lrcorner}$. Then $\Delta$ is Sidon when- 
ever one of the following holds:

(i ) $W l^{\infty}(\Delta)=M(G)^{\wedge} \mid \Delta$,

(ii) $W c_{0}(\Delta)=L^{1}(G)^{\wedge} \mid \Delta$,

(iii) $W C_{\Delta}(G)^{\wedge} \mid \Delta=l^{1}(\Delta)$,

(iv) $W L_{\Delta}^{\infty}(G)^{\wedge} \mid \Delta=l^{1}(\Delta)$.

Proof. (i) Taking the Dirac measure at $e$ we see $1 \in W l^{\infty}(\Delta)$. Thus $l^{\infty}(\Delta) \leqq W l^{\infty}(\Delta) \leqq l^{\infty}(\Delta)$ hence $l^{\infty}(\Delta)=W l^{\infty}(\Delta)=M(G)^{\wedge} \mid \Delta$ so $\Delta$ is Sidon.

(ii) By hypothesis we cannot have $W(\chi)=0$ for any $\chi \in \Delta$, so $W^{-1} L^{1}(G)^{\wedge} \mid \Delta=c_{0}(\Delta)$. Now in 1.12 we take $A \equiv c_{0}(\Delta)$ with norm $\|\cdot\|_{\infty}$, $\alpha$ the canonical injection, $B \equiv L^{1}(G)$ with norm $\|\cdot\|_{1}, \beta(\hat{f})=\hat{f} \mid \Delta$ and $\psi \equiv W^{-1}$. The hypotheses are readily verified so we conclude that $\left\|W^{-1}\right\|_{\infty}<\infty$. Applying 1.10, whenever $t \in T_{\Delta}(G)$,

$$
\sum_{\chi \in \Delta}|\hat{t}(\chi)| \leqq\left\|W^{-1}\right\|_{\infty} \kappa\|t\|_{\infty} \text {. }
$$

So $\Delta$ is Sidon.

(iii) Again, $W$ is never zero so we may apply 1.12 taking $A \equiv C_{\Delta}(G), B \equiv l^{1}(\Delta), \alpha(f)=\widehat{f} \mid \Delta, \beta$ the canonical injection and $\psi \equiv W^{-1}$. As in (ii) we deduce that $\Delta$ is Sidon.

(iv) Apply the same method as (iii).

Note. The converse to each of these assertions is false. Even if $\Delta$ is replaced by $\Delta_{0} \equiv\{\chi \in \Delta: W(\chi) \neq 0\}$ and $\Delta_{0}$ is Sidon, these inclusions are strict if $\Delta_{0}$ is infinite and $W \in c_{0}(\Delta)$.

THeOREM 1.15. Let $\Delta \subseteq X, W \in \mathbb{C}^{\Delta}$ and $\Delta_{0}$ be as above. Assuming the constants in (ii), (iii) and (iv) to be the least possible, these are equivalent:

(i) $\Delta_{0}$ is Sidon with constant $\kappa$,

(ii) $f \in L_{\Delta_{0}}^{\infty}(G)$ implies $\sum_{\chi \in \Delta} W(\chi) \hat{f}(\chi) \chi \in A_{\Delta_{0}}(G)$ and $\|W \hat{f}\|_{1} \leqq$ $\kappa\left\|\sum_{x \in \Delta} W(\chi) \hat{f}(\chi) \chi\right\|_{\infty}$,

(iii) $t \in T_{\Delta_{0}}(G)$ implies $\|W \hat{t}\|_{1} \leqq \kappa\left\|\sum_{x \in \Delta} W(\chi) \hat{t}(\chi) \chi\right\|_{\infty}$, and

(iv) for all $\phi \in l^{\infty}\left(\Delta_{0}\right)$ there is $\mu \in M(G)$ such that $\hat{\mu} \mid \Delta_{0}=W_{\phi}$ and $\|\mu\| \leqq \kappa\|W \dot{\phi}\|_{\infty}$.

Proof. (i) $\Rightarrow$ (ii). If $f \in L_{\Delta_{0}}^{\infty}(G)$ then

$$
\|W \widehat{f}\|_{1}=\sum_{\chi \in \Delta_{0}}|W(\chi) \hat{f}(\chi)| \leqq\|W\|_{\infty}\|\hat{f}\|_{1}
$$

so that if $\Delta_{0}$ is Sidon, (ii) follows.

(ii) $\Rightarrow$ (iii) is obvious.

(iii) $\Rightarrow$ (i). If $t \in T_{\Delta_{0}}(G)$ define $u \in T_{\Delta_{0}}(G)$ by taking 


$$
\widehat{u}(\chi)=W^{-1}(\chi) \hat{t}(\chi) \text { for all } \chi \in \Delta_{0} \text {. }
$$

They by (iii),

$$
\sum_{\chi \in \Delta_{0}}|\hat{t}(\chi)|=\sum_{\chi \in \Delta_{0}}|W(\chi) \hat{u}(\chi)| \leqq \kappa\left\|\sum_{\chi \in \Delta_{0}} W(\chi) \hat{u}(\chi)\right\|_{\infty} \leqq \kappa\|t\|_{\infty}
$$

so (i) follows.

(i) $\Rightarrow$ (iv). If $\phi \in l^{\infty}(\Delta)$ and $W \in \mathfrak{M}(\Delta)$ then $\phi W \in l^{\infty}(\Delta)$ hence (iv) results from (i) and 1.11.

(iv) $\Rightarrow$ (i). If $\psi \in l^{\infty}\left(\Delta_{0}\right)$ and $\Phi \in \mathfrak{F}\left(\Delta_{0}\right)$ let

$$
\psi_{\Phi}(\chi)=\left\{\begin{array}{l}
W^{-1}(\chi) \psi(\chi) \text { if } \chi \in \Phi \\
0 \text { if } \chi \in \Delta_{0} \mid \Phi
\end{array} \text {, so that } \psi_{\Phi} \in c_{0}\left(\Delta_{0}\right) .\right.
$$

By hypothesis there is $\mu_{\phi} \in M(G)$ with $\hat{\mu}_{\phi} \mid \Delta_{0}=W \psi_{\phi}$ and

$$
\left\|\mu_{\Phi}\right\| \leqq \kappa\left\|W \psi_{\Phi}\right\|_{\infty} \leqq \kappa\|\psi\|_{\infty} \text {. }
$$

Thus $\left\{\mu_{\Phi}: \Phi \in \mathfrak{F}(\Delta)\right\}$ is bounded in $M(G)$ hence by Alaoglu's theorem it has a weakly convergent subnet. So there is $\mu \in M(G)$ with $\hat{\mu} \mid \Delta_{0}=\psi$, and $\Delta_{0}$ must be Sidon.

1.16. Many characterisations of Sidon sets have weighted analogues, like 1.10. More of these may be found in [11].

\section{Thick $W$-Sidon sets.}

2.0. To find $W$-Sidon sets which are not Sidon it suffices, by 1.2 , to take $\Delta \subseteq X$ not Sidon and then choose $W \in l^{2}(\Delta)$ (such $\Delta$ exist since infinite subgroups are not Sidon). It is the purpose of this section to exhibit non-Sidon sets $\Delta$ which are $W$-Sidon for some $W \notin l^{2}(\Delta)$. These sets are in the dual of the circle group and are not even $\Lambda(1)$.

The proof relies on Riesz products and therefore requires a sort of independence condition on $\Delta$. Recall $\Delta^{2}=\{\chi \xi: \chi, \xi \in \Delta\}$ whenever $\Delta \subseteq X$.

Theorem 2.1. Suppose $\Delta=\bigcup\left\{\Delta_{n}: n \in \mathfrak{R}\right\}$ where $0<\nu\left(\Delta_{n}\right)<\aleph_{0}$ and

(i) $1 \notin \Delta_{0}$,

(ii) $\Delta_{n}^{-1}=\Delta_{n}$,

(iii) $\Delta_{n+1} \subseteq X \backslash \cup\left\{\Delta_{0}^{\varepsilon_{0}} \Delta_{1}^{\varepsilon_{1}} \cdots \Delta_{n}^{\varepsilon_{n}}: \varepsilon_{i} \in\{0,1,2\}\right.$ for $0 \leqq i \leqq n$ and at most one $\varepsilon_{i}$ equal to 2$\}$, and

(iv) $\Delta_{n+1}^{2} \subseteq X \backslash \cup\left\{\Delta_{0}^{\varepsilon_{0}} \Delta_{1}^{\varepsilon_{1}} \cdots \Delta_{n}^{\varepsilon_{n}}: \varepsilon_{i} \in\{0,1\} \quad\right.$ for $\quad 0 \leqq i \leqq n$ and $\left.\sum_{i=0}^{n} \varepsilon_{i} \geqq 1\right\}$ 
Define $W: \Delta \rightarrow(0,1]$ to equal $\nu\left(\Delta_{n}\right)^{-1}$ on $\Delta_{n}$. The conclusion is that $\Delta$ is W-Sidon.

Proof. Suppose $\phi \in \mathfrak{R}^{\Delta}$ with $\|\phi\|_{\infty} \leqq 1$. For $n \in \mathfrak{R}$ define $t_{n} \in T(G)$ by

$$
t_{n}=\left(2 \nu\left(\Delta_{n}\right)\right)^{-1}\left(\sum_{\substack{\chi \in A_{n} \\ x^{2} \neq 1_{n}}} \phi(\chi)(\chi+\bar{\chi})+\sum_{\substack{\chi \in A_{n} \\ x^{2}=1^{n}}} \phi(\chi) \chi\right) .
$$

It is easy to see that

$$
t_{n} \text { is real-valued }
$$

$$
\left\|t_{n}\right\|_{\infty} \leqq 1
$$

$$
\text { and, by (ii), } \hat{t}_{n}(\chi)=\left\{\begin{array}{l}
\left(2 \nu\left(\Delta_{n}\right)\right)^{-1} \dot{\phi}(\chi) \text { if } \chi \in \Delta_{n} \\
0 \text { if } \chi \in X \backslash \Delta_{n}
\end{array}\right. \text {. }
$$

Next for $N \in \Re$ set $P_{N}=\prod_{n=0}^{N}\left(1+t_{n}\right)$ so that $P_{N}=1+\sum_{n=0}^{N} t_{n}+Q_{N}$ where

$$
\begin{aligned}
Q_{N}= & \sum_{0 \leqq n_{1}<n_{2} \leqq N} t_{n_{1}} t_{n_{2}}+\sum_{0 \geqq_{1}<n_{2} \geqq_{3} \leqq N} t_{n_{1}} t_{n_{2}} t_{n_{3}}+\cdots \\
& +t_{0} t_{1} \cdots t_{N} .
\end{aligned}
$$

$$
\text { Now } \hat{P}_{N}\left|\Delta_{n}=\hat{t}_{n}\right| \Delta_{n} \text { if } 0 \leqq n \leqq N
$$

provided that whenever $0 \leqq n \leqq N$,

$$
\Delta_{n} \subseteq X \backslash\left[\operatorname{sp}(\mathbf{1}) \cup \cup\left\{\Delta_{m}: 0 \leqq m \leqq N \text { and } m \neq n\right\} \cup s p\left(Q_{N}\right)\right] .
$$

Consequently the lemma to follow ensures this for each $N \in \Re$.

By (2.1.1), (2.1.2) and (2.1.3), for each $N$, if we have

$$
1 \notin \bigcup\left\{A_{n}: 0 \leqq n \leqq N\right) \cup \operatorname{sp}\left(Q_{N}\right)
$$

then

$$
\left\|P_{N}\right\|_{1}=\int_{G} P_{N}=1+\sum_{n=0}^{N} \int_{G} t_{n}+\int_{G} Q_{N}=1 .
$$

Again, the lemma assures us of this.

So by (2.1.6), $\left\{P_{N}: N \in \mathfrak{R}\right\}$ is bounded in $M(G)$ and thus has a weak cluster point $\tau \in M(G)$; let $\mu=2 \tau$. Then for each $n \in \mathfrak{R}$ and $\chi \in \Delta_{n}$,

$$
\begin{aligned}
\hat{\mu}(\chi)=2 \hat{\tau}(\chi) & =2 \hat{t}_{n}(\chi) \text { by }(2.1 .5) \\
& =\nu\left(\Delta_{n}\right)^{-1} \phi(\chi) \text { by }(2.1 .3) \\
& =W(\chi) \phi(\chi) \text { by definition of } W .
\end{aligned}
$$

Thus $\hat{\mu} \mid \Delta=W \phi$ so by 1.13 (iii), $\Delta$ is $W$-Sidon. 
LEMMA 2.2. Suppose $\left\{\Delta_{n}: n \in \mathfrak{R}\right\} \subseteq \mathfrak{B}(X)$ satisfies conditions (i) to (iv) of the previous theorem. Then with $Q_{N}$ given by (2.1.4), for each $N \in \mathfrak{R}$,

(i) $0 \leqq n \leqq N$ implies

$$
\Delta_{n} \leqq X \backslash\left\{\{1\} \cup \cup\left\{\Delta_{m}: 0 \leqq m \leqq N \text { and } m \neq n\right\} \cup s p\left(Q_{N}\right)\right] \text {, and }
$$

(ii) $1 \notin \bigcup\left\{\Delta_{n}: 0 \leqq n \leqq N\right\} \cup s p\left(Q_{N}\right)$.

Proof. By (2.1.4) and (2.1.3),

$$
s p\left(Q_{N}\right) \subseteq \bigcup\left\{\Delta_{0}^{\varepsilon_{0}} \Delta_{1}^{\varepsilon_{1}} \cdots \Delta_{N}^{\varepsilon_{N}}: \varepsilon_{i} \in\{0,1\} \text { for } 0 \leqq i \leqq N \text { and } \sum_{i=0}^{N} \varepsilon_{i} \geqq 2\right\} \text {. }
$$

For brevity define

$$
A(N, n)=\{1\} \cup \cup\left\{\Delta_{m}: 0 \leqq m \leqq N \text { and } m \neq n\right\} \text { for } 0 \leqq n \leqq N,
$$

and

$$
B(N, j)=\bigcup\left\{\Delta_{0}^{\varepsilon_{0}} \Delta_{1}^{\varepsilon_{1}} \cdots \Delta_{N}^{\varepsilon}: \varepsilon_{i} \in\{0,1\} \text { and } \sum_{i=0}^{N} \varepsilon_{i} \geqq j\right\} \text { for } j \in\{1,2\} .
$$

In these terms we have to prove, for each $N \in \Re, 0 \leqq n \leqq N$ implies $\Delta_{n} \subseteq X \backslash[A(N, n) \cup B(N, 2)]$, and

$$
1 \notin \bigcup\left\{\Delta_{n}: 0 \leqq n \leqq N\right\} \cup B(N, 2) .
$$

A straightforward induction, relying heavily on 2.1(ii), completes the argument.

THEOREM 2.3. There is a subset $\Delta$ of 3 which is W-Sidon for some $W \in l^{\infty}(\Delta) \backslash l^{2}(\Delta)$ yet which is not $\Delta(1)$.

Proof. Take $m_{0} \neq 0$ and let $\Delta_{0}=\left\{ \pm m_{0}\right\}$. Supposing $\Delta_{0}, \cdots \Delta_{n}$ have been defined so as to satisfy the hypotheses of 2.1 , let $m \in \mathfrak{N}$ be the supremum of the finite set

$$
\bigcup\left\{\varepsilon_{0} \Delta_{0}+\cdots+\varepsilon_{n} \Delta_{n}: \varepsilon_{i} \in\{0,1,2\} \text { with at most one } \varepsilon_{i}=2\right\} \text {. }
$$

Now if $n=0$ set $A_{1}=\{ \pm(m+1)\}$ and if $n \geqq 1$ take

$$
\Delta_{n+1}=\{ \pm j(m+1): 1 \leqq j \leqq[(n+1) / 2]\} \text {. }
$$

Since $\Delta_{n+1}+\Delta_{n+1}$ is also disjoint from the finite set above, it is disjoint from

$$
\bigcup\left\{\varepsilon_{0} \Delta_{0}+\cdots+\varepsilon_{n} \Delta_{n}: \varepsilon_{i} \in\{0,1\} \text { with } \sum_{i=0}^{n} \varepsilon_{i} \geqq 1\right\} .
$$

Consequently 2.1 shows $\Delta \equiv \bigcup\left\{\Delta_{n}: n \in \mathfrak{R}\right\}$ is W-Sidon where 


$$
\sum_{\chi \in \Delta}|W(\chi)|^{2} \geqq \sum_{n \in \Re}(1+n)^{-1}=\infty
$$

so $W \notin l^{2}(\Delta)$.

By construction $\Delta$ contains arbitrarily long arithmetic progressions hence it is not $\Lambda(1)$ by [9], (4.1).

2.4. Using multiplier notation from 4.2 , by 3.3 to follow,

$$
l^{2}(\Delta)=\left(C_{\Delta}(G), A_{\Delta}(G)\right)
$$

whenever $\Delta$ is a subgroup of $X$. If $\Delta \subseteq X$, Parseval's identity shows

$$
l^{2}(\Delta) \subseteq\left(C_{\Delta}(G), A_{\Delta}(G)\right) .
$$

To find $\Delta$ for which this inclusion is strict it suffices to take $\Delta$ an infinite Sidon set so that $1 \in\left(C_{\Delta}(G), A_{\Delta}(G)\right) \backslash l^{2}(\Delta)$. However 2.3 provides examples of non-Sidon sets $\Delta$ in 3 for which the strict inclusion holds. It also indicates the impossibility of extending [1], Theorem 1 to arbitrary subsets of $X$.

\section{The algebra of weight functions.}

3.0. From 1.10 we may read off more expressions for $\|W\|_{4}$ :

$$
\begin{aligned}
\|W\|_{\Delta} & =\sup \left\{\sum_{\chi \in \Delta}|W(\chi) \hat{f}(\chi)|: f \in C_{\Delta}(G) \text { with }\|f\|_{\infty} \leqq 1\right\} \\
& =\sup \left\{\inf \left\{\|f\|_{1}: f \in L^{1}(G) \text { with } \hat{f} \mid \Delta=W \phi\right\}: \phi \in c_{0}(\Delta) \text { and }\|\phi\|_{\infty} \leqq 1\right\} \\
& \left.=\sup \{\inf \|\mu\|: \mu \in M(G) \text { with } \hat{\mu} \mid \Delta=W \phi\}: \phi \in l^{\infty}(\Delta) \text { and }\|\phi\|_{\infty} \leqq 1\right\} .
\end{aligned}
$$

THEOREM 3.1. $\mathfrak{W}(\Delta)$ is a commutative Banach algebra under $\|\cdot\|_{\Delta}$ and pointwise operations. It has an identity iff $\Delta$ is Sidon.

Proof. The following straightforward formulae establish that $\|\cdot\|_{\Delta}$ makes $\mathfrak{W}(\Delta)$ into a commutative normed algebra under pointwise operations.

Suppose $W_{1}, W_{2} \in \mathfrak{W}(\Delta), \alpha \in \mathbb{E}$ and $t \in T_{\Delta}(G)$ with $\|t\|_{\infty} \leqq 1$. Then

$$
\begin{aligned}
& \sum_{\gamma \in \Delta}\left|\left(W_{1}(\chi)+W_{2}(\chi)\right) \hat{t}(\chi)\right| \leqq \sum_{\chi \in \Delta}\left|W_{1}(\chi) \hat{t}(\chi)\right|+\sum_{\chi \in \Delta}\left|W_{2}(\chi) \hat{t}(\chi)\right| \\
& \leqq\left\|W_{1}\right\|_{\Delta}+\left\|W_{2}\right\|_{\Delta} ; \\
& \sum_{\chi \in \Delta}\left|\alpha W_{1}(\chi) \hat{t}(\chi)=\right| \alpha\left|\sum_{\chi \in \Delta}\right| W_{1}(\chi) \hat{t}(\chi)|\leqq| \alpha \mid\left\|W_{1}\right\|_{\Delta} ; \\
& \sum_{\chi \in \Delta}\left|W_{1}(\chi) W_{2}(\chi) \hat{t}(\chi)\right| \leqq\left\|W_{1}\right\|_{\infty} \sum_{\chi \in \Delta}\left|W_{2}(\chi) \hat{t}(\chi)\right| \leqq\left\|W_{1}\right\|_{\Delta}\left\|W_{2}\right\|_{\Delta} \text { by } 1.1 ;
\end{aligned}
$$

and if $\|W\|_{\Delta}=0$ then $\|W\|_{\infty}=0$ hence $W=\mathbf{0}$.

Suppose $\left\{W_{n}: n \in \mathfrak{R}\right\} \subseteq \mathfrak{W}(\Delta)$ is a Cauchy sequence. Then by 1.1 again, $\left\|W_{n}-W_{m}\right\|_{\infty} \rightarrow 0$ hence there is $W \in l^{\infty}(\Delta)$ for which $\left\|W-W_{n}\right\|_{\infty} \rightarrow 0$. 
If $\varepsilon>0$, there is $N \in \mathfrak{R}$ such that $n \geqq N$ implies, for all $t \in T_{\Delta}(G)$ with $\|t\|_{\infty} \leqq 1$,

$$
\sum_{\chi \in \Delta}\left|\left(W_{n}(\chi)-W_{m}(\chi)\right) \hat{t}(\chi)\right|<\varepsilon .
$$

Letting $m \rightarrow \infty$, the same inequality holds with $W$ replacing $W_{m}$. So $n \geqq N$ implies $\left\|W_{n}-W\right\|_{\Lambda}<\varepsilon$. Furthermore

$$
\|W\|_{\Delta}-\left\|W_{N}\right\|_{\Delta} \leqq\left\|W-W_{N}\right\|_{\Delta}<\varepsilon
$$

hence $\|W\|_{\Delta}<\varepsilon+\left\|W_{N}\right\|_{\Delta}<\infty$. Thus $W_{n} \rightarrow W$ in $\mathfrak{W}(\Delta)$.

Finally $\mathfrak{W}(\Delta)$ has an identity iff $1 \in \mathfrak{W}(\Delta)$ iff $\Delta$ is Sidon.

3.2. From 1.1 we have: $\Delta$ is Sidon iff $\mathfrak{B}(\Delta)=l^{\infty}(\Delta)$. Our next few results consider $\mathfrak{W}(\Delta)$ contained in $c_{0}(\Delta)$.

THEOREM. If $L^{1}(G)^{\wedge} \mid \Delta \cong \mathfrak{W}(\Delta)$ (in particular, if $\mathfrak{W}(\Delta)=c_{0}(\Delta)$ ) then $\Delta$ is Sidon.

Proof. Suppose $f \in C_{\Delta}(G)$-we show $\|\hat{f}\|_{1}<\infty$ by using the boundedness principle 1.11. Take therein $A \equiv l^{1}(\Delta)$ with $\alpha$ the identity, $B \equiv L^{1}(G)$ with $\beta(g)=\hat{f} \hat{g} \mid \Delta$ and $C \equiv \mathbb{C}^{\Delta}$ with the product topology. Then for some constant $\kappa$, for all $g \in L^{1}(G)$, there is $\phi \in l^{1}(\Delta)$ such that $\dot{\phi}=\hat{f} \hat{g} \mid \Delta$ and $\sum_{\chi \in \Delta}|\phi(\chi)| \leqq \kappa\|g\|_{1}$. In other words, $\sum_{\chi \in \Delta}|\hat{f}(\chi) \hat{g}(\chi)| \leqq$ $\kappa\|g\|_{1}$.

Allowing $g$ to vary over an approximate identity,

$$
\sum_{\chi \in \Delta}|\hat{f}(\chi)|<\infty
$$

as required.

3.3. At the other end of the spectrum we can have equality in 1.2 .

TheOREM. If $\Delta$ is a subgroup of $X$ then $\mathfrak{W}(\Delta)=l^{2}(\Delta)$.

Proof. Obviously $l^{2}(\Delta) \subseteq \mathfrak{W}(\Delta)$ by 1.2 .

If $W \in \mathfrak{M}(\Delta)$ then by 1.3 we may suppose $\Delta=X$. Now by 1.10(iii) and [1], 2.1(a), it follows that $W \in l^{2}(\Delta)$. This completes the proof.

REMARKS 3.4. From 3.3 it follows that if $\Delta$ is cofinite in some subgroup of $X$ then $\mathfrak{W}(\Delta)=l^{2}(\Delta)$.

Similarly by [10], 8.7.8, if $\Delta$ is cofinite in the positive cone of the ordered dual of a compact connected abelian group then $\mathfrak{B}(\Delta)=$ $l^{2}(\Delta)$. 
THEOREM 3.5. For $\Delta \subseteq X, \mathfrak{W}(\Delta)$ is an ideal in $M(G)^{\wedge} \mid \Delta$ which is improper iff $\Delta$ is Sidon. For each $W \in \mathfrak{W}(\Delta),\|W\|_{\diamond} \leqq\|W\|_{\Delta}$ (see 1.6 for notation).

Proof. If $W \in \mathfrak{B}(\Delta)$ by applying $1.10(\mathrm{iv})$ to $\phi=1$, there is $\nu \in M(G)$ with $\hat{\nu} \mid \Delta=W$ and $\|\nu\| \leqq\|W\|_{\Delta}$. So $\mathfrak{W}(\Delta) \subseteq M(G)^{\wedge} \mid \Delta$ and for all $W \in \mathfrak{W}(\Delta),\|W\|_{o} \leqq\|W\|_{\Delta}$.

Obviously the algebraic operations on these spaces coincide and if $\mu \in M(G)$, for all $t \in T_{\Delta}(G)$ with $\|t\|_{\infty} \leqq 1$,

$$
\sum_{\chi \in \Delta}|W(\chi) \hat{\mu}(\chi) \hat{t}(\chi)| \leqq\|\hat{\mu}\|_{\infty}\|W\|_{\Delta} .
$$

Thus $W \hat{\mu} \mid \Delta \in \mathfrak{W}(\Delta)$ which, by 3.1 , is consequently an ideal in $M(G)^{\wedge} \mid \Delta$ which is improper iff $\Delta$ is Sidon.

Note. By 3.3, $\mathfrak{W}(\Delta)$ need not be closed in $M(G)^{\wedge} \mid \Delta$.

3.6. As algebras, for $\Delta \subseteq X$,

$$
l^{2}(\Delta) \subseteq \mathfrak{W}(\Delta) \subseteq M(G)^{\wedge} \mid \Delta \cong l^{\infty}(\Delta) .
$$

Each is endowed with a norm-they are $\|\cdot\|_{2},\|\cdot\|_{\Delta},\|\cdot\|_{\delta}$ and $\|\cdot\|_{\infty}$ respectively. When $\Delta$ is a subgroup of $X,\|\cdot\|_{2}$ and $\|\cdot\|_{\Delta}$ are actually equivalent (by 3.3 and the open mapping theorem or [1], $(2.1)(b)$ ) on $\mathfrak{W}(\Delta)$.

A different proof of the inequality $\|\cdot\|_{\delta} \leqq\|\cdot\|_{\Delta}$ (established above) follows by the method in [10], 1.9.1 which yields the characterisation: for $W \in \mathfrak{M}(\Delta)$,

$$
\|W\|_{\delta}=\sup \left\{\left|\sum_{\chi \in \Delta} W(\chi) \hat{t}(\chi)\right|: t \in T_{\Delta}(G) \text { and }\|t\|_{\infty} \leqq 1\right\} .
$$

This shows why, in 1.0, we kept the modulus signs inside the sum. We now consider when pairs of these norms are equivalent.

THEOREM 3.7. For $\Delta \subseteq X$ these are equivalent:

(i) $\Delta$ is Sidon,

(ii) $\|\cdot\|_{\infty}$ and $\|\cdot\|_{\Delta}$ are equivalent on $\mathfrak{W}(\Delta)$,

(iii) $\|\cdot\|_{\delta}$ and $\|\cdot\|_{\Delta}$ are equivalent on $M(G)^{\wedge} \mid \Delta$,

(vi) $\|\cdot\|_{o}$ and $\|\cdot\|_{\infty}$ are equivalent on $M(G)^{\wedge} \mid \Delta$.

Proof. (a) If $\Delta$ is Sidon and $W \in \mathfrak{S}(\Delta)$ and $t \in T_{\Delta}(G)$ with $\|t\|_{\infty} \leqq 1$ then

$$
\sum_{\chi \in \Delta}|W(\chi) \hat{t}(\chi)| \leqq\|W\|_{\infty} \sum_{\chi \in \Delta}|\hat{t}(\chi)| \leqq\|W\|_{\infty}\|\mathbf{1}\|_{\Delta}
$$

Thus whenever $W \in \mathfrak{S}(\Delta)=M(G)^{\wedge} \mid \Delta$, 


$$
\|W\|_{\infty} \leqq\|W\|_{\delta} \leqq\|W\|_{\Delta} \leqq\|\mathbf{1}\|_{\Delta}\|W\|_{\infty} \leqq\|\mathbf{1}\|_{\perp}\|W\|_{\delta},
$$

so the norms are pairwise equivalent.

(b) If $\Delta$ is not Sidon then by $3.2, l^{2}(\Delta) \cong \mathfrak{B}(\Delta) \subset c_{0}(\Delta)$. Since $l^{2}(\Delta)$ contains all finite linear combinations of characteristic functions of singleton subsets of $\Delta$ and these are dense in $c_{0}(\Delta), \mathfrak{S}(\Delta)$ cannot be closed in $c_{0}(\Delta)$. Thus $\mathfrak{B}(\Delta)$ cannot be complete under the restriction of $\|\cdot\|_{\infty}$. So by $3.1,\|\cdot\|_{\infty}$ and $\|\cdot\|_{\Delta}$ cannot be equivalent on $\mathfrak{W}(\Delta)$.

(c) If $\|\cdot\|_{\delta}$ and $\|\cdot\|_{\Delta}$ are equivalent on $M(G)^{\wedge} \mid \Delta$ then $\mathfrak{B}(\Delta)=$ $M(G)^{\wedge} \mid \Delta$ hence by $3.5, \Delta$ is Sidon.

(d) If $\|\cdot\|_{\delta}$ and $\|\cdot\|_{\infty}$ are equivalent on $M(G)^{\wedge} \mid \Delta$ then it is complete under $\|\cdot\|_{\infty}$ and hence $c_{0}(\Delta) \subseteq M(G)^{\wedge} \mid \Delta$. So by 1.9(ii), $C_{-}(G)^{\wedge} \mid \Delta \leqq l^{1}(\Delta)$ and so $\Delta$ is Sidon.

REMARKS 3.8. (i ) As a Banach algebra, $\mathfrak{W}(\Delta)$ is neither separable nor a $B^{*}$-algebra in general. The former follows by 1.1 and the latter by 3.3 .

(ii) Considering $C_{\Delta}(G)^{\wedge} \mid \Delta$ as a sequence space, $\mathfrak{B}(\Delta)$ is its $\alpha$-dual (see [8], §30). However 3.3 shows that $C_{\Delta}(G)^{\wedge} \mid \Delta$ is not, in general, a perfect sequence space.

3.9. Refer to [4], 1.1 for the definition of a $p$-Sidon set.

Theorem. Let $\Delta \leqq X$ and $1 \leqq p<2$. Then $\Delta$ is $p$-Sidon iff $l^{p^{\prime}}(\Delta) \subseteq \mathfrak{W}(\Delta)$.

Proof. For $p=1$ this is just 1.1 (it is trivial when $p=2$ ). If $1<p<2$ and $\Delta$ is $p$-Sidon then by [4], 1.2(ii), $f \in C_{A}(G)$ implies $\hat{f} \mid \Delta \in l^{p}(\Delta)$. So if $W \in l^{p^{\prime}}(\Delta)$, Hölder's inequality shows

$$
\sum_{\chi \in\lrcorner}|W(\chi) \hat{f}(\chi)|<\infty
$$

hence by $1.10, W \in \mathfrak{M}(\Delta)$.

Conversely if $l^{p^{\prime}}(\Delta) \cong \mathfrak{W}(\Delta)$ then by $3.5, l^{p^{\prime}}(\Delta) \subseteq M(G)^{\wedge} \mid \Delta$. So by [4], 1.2(iv), $\Delta$ is $p$-Sidon.

From this follows, by the Hausdorff-Young theorem, a converse of 3.2 for $p>1$.

COROLLARY. If $1<p<2$ and $\Delta$ is $p$-Sidon then $L^{p}(G)^{\wedge} \mid \Delta \subseteq \mathfrak{W}(\Delta)$.

4. Multipliers and $W$-Sidon sets.

4.0. When $\Delta$ is Sidon, spaces of $\Delta$-spectral functions collapse. Not only is $L_{\lrcorner}^{\infty}(G)=A_{\lrcorner}(G)$ but $M_{\lrcorner}(G)=\bigcap\left\{L_{\lrcorner}^{p}(G): 1 \leqq p<\infty\right\}$. In this 
section we investigate analogues for $W$-Sidon sets.

In this context it is natural to consider the trigonometric series $\sum_{\chi \in \Delta} W(\chi) \hat{\mu}(\chi) \chi$ for $\mu \in M_{\Delta}(G)$ (see for instance 1.15.) To ensure such objects make sense we define, for $\Delta \subseteq X$,

$$
T: l^{\infty}(\Delta) \times P M_{\Delta}(G) \longrightarrow P M_{\Delta}(G)
$$

by

$$
T(\phi, \pi)=\sum_{\chi \in d} \phi(\chi) \hat{\pi}(\chi) \chi
$$

When $\phi$ is fixed we shall use the single variable notation $T_{\dot{\phi}}$ even for its restriction to some subset of $P M_{\Delta}(G)$.

If $\phi \in l^{\infty}(\Delta)$ let $\pi_{\phi} \in P M_{\Delta}(G)$ be given by

$$
\hat{\pi}_{\phi}(\chi)=\left\{\begin{array}{ll}
\dot{\phi}(\chi) & \text { if } \chi \in \Delta \\
0 & \text { if } \chi \in X \backslash \Delta
\end{array} .\right.
$$

Then $T(\phi, \pi)=\pi_{\dot{\phi}} * \pi$, for all $\pi \in P M_{\Delta}(G)$, so $T$ is just convolution from $P M_{\Delta}(G) \times P M_{\Delta}(G)$ into $P M_{\Delta}(G)$. From this it is evident that $T$ is bilinear, continuous and behaves nicely under translation and convolution.

THEOREM 4.1. If $\Delta$ is W-Sidon and $t \in T_{\Delta}(G)$ then

$$
\left\|T_{W} t\right\|_{p} \leqq 2\|W\|_{\Delta} p^{1 / 2}\|t\|_{2} \text { if } 2<p<\infty
$$

and

$$
\left\|T_{W} t\right\|_{2} \leqq 8\|W\|_{\Delta}\|t\|_{1}
$$

Proof. We modify Rudin's proof for Sidon sets. For an exposition of the Rademacher functions $\left\{r_{n}: n \in \mathfrak{N}\right\}$ refer to [2], Chapter 14. By redefining $r_{n}$ on a set of measure zero so that is is right continuous at each dyadic rational and left continuous at 1 , we ensure $r_{n} \in\{ \pm 1\}^{[0,1]}$.

For $t \in T_{\Delta}(G)$ let $j \in X^{\Re}$ be an injection with $s p(t) \cong j(\Re)$, and define $R: X \rightarrow\{ \pm 1\}^{[0,1]}$ by

$$
R_{\chi}=\left\{\begin{array}{ll}
r_{\jmath}^{-1}(\chi) & \text { if } \chi \in j(\Re) \\
r_{0} & \text { if } \chi \in X \backslash j(\Re)
\end{array} .\right.
$$

Now let $f: G \times[0,1] \rightarrow$ c be given by

$$
f(x, \rho)=\sum_{\chi \in X} \hat{t}(\chi) R_{\chi}(\rho) \chi(x) .
$$

Using single variable notation we have $f_{\rho} \in T_{\Delta}(G)$ for all $\rho \in[0,1]$ and for all $x \in G, f_{i x}=\sum_{n \in \mathfrak{N}} \hat{t}(j(n)) j(n)(x) r_{n}$ which is a Rademacher series. 
Since $f$ is a finite sum of functions which are measurable on $G \times[0,1]$ each dominated by the constant $\|t\|_{\infty}, f$ is integrable and we may use Fubini's theorem.

Suppose $\rho \in[0,1]$. By $1.10(\mathrm{iv})$, there is $\mu_{\rho} \in M(G)$ such that $\hat{\mu}_{\rho}(\chi)=W(\chi) R_{\chi}(\rho)$, for all $\chi \in \Delta$ and $\left\|\mu_{\rho}\right\| \leqq\|W\|_{\Delta} \| R$. $(\rho)\left\|_{\infty}=\right\| W \|_{\Delta}$. So for $\chi \in \Delta$,

$$
\hat{\mu}_{\rho}(\chi) \hat{f}_{\rho}(\chi)=W(\chi) R_{\chi}(\rho) \hat{t}(\chi) R_{\chi}(\rho)=W(\chi) \hat{t}(\chi)=\left(T_{W} t\right)^{\wedge}(\chi):
$$

and if $\chi \in X \backslash \Delta$,

$$
\left(T_{W} t\right)^{\wedge}(\chi)=0=\hat{f}_{\rho}(\chi) .
$$

Thus $T_{W} t=\mu_{\rho} * f_{\rho}$ hence $\left\|T_{W} t\right\|_{p} \leqq\left\|\mu_{\rho}\right\|\left\|f_{\rho}\right\|_{p} \leqq\|W\|_{\Delta}\left\|f_{\rho}\right\|_{p}$.

So when $p=2 m$ (for some $m \in \mathfrak{N}$ ),

$$
\int_{G}\left|T_{W} t\right|^{2 m} \leqq\|W\|_{\Delta}^{2 m} \int_{G}\left|f_{\rho}\right|^{2 m}
$$

But a property of Rademacher series ([2], 14.2.1) ensures that for all $x \in G$,

$$
\int_{0}^{1}\left|f_{x}\right|^{2 m} \leqq(4 m)^{m}\left(\sum_{\chi \in X}|\hat{t}(\chi) \chi(x)|^{2}\right)^{m} .
$$

So using Fubini's theorem to integrate (4.1.3) along $[0,1]$,

$$
\int_{G}\left|T_{W} t\right|^{2 m} \leqq\|W\|_{\Delta}^{2 m}(4 m)^{m}\left(\sum_{\chi \in \Delta}|\hat{t}(\chi)|^{2}\right)^{m} .
$$

Now given any $p \in(2, \infty)$ choose $m \in \mathfrak{R}$ such that $2(m-1)<p \leqq 2 m$ and $1<m \leqq p$. Then (4.1.4) guarantees

$$
\left\|T_{W} t\right\|_{p} \leqq\left\|T_{W} t\right\|_{2 m} \leqq 2\|W\|_{4} m^{1 / 2}\|t\|_{2} \leqq 2\|W\|_{\Delta} p^{1 / 2}\|t\|_{2}
$$

which yields (4.1.1).

To prove (4.1.2) we argue similarly, except that for $t \in T_{\Delta}(G)$ we redefine $f(x, \rho)=\sum_{\chi \in\lrcorner} W(\chi) \hat{t}(\chi) R_{\chi}(\rho) \chi(x)$.

Notation 4.2. When $E, F \subseteq P M(G)$ and $\Delta \leqq X$ we shall write $\left(E_{\Delta}, F_{\Delta}\right)$ for the set of all $\phi \in \mathbb{C}^{\Delta}$ such that $\pi \in E_{\Delta}$ implies $\phi \hat{\pi}\left|\Delta \in F_{\Delta}\right| \Delta$. Writing $(E, F)$ for $\left(E_{X}, F_{X}\right)$ we return to the standard multiplier notation.

4.3. Exploiting the conclusions of 4.1 we have

THEOREM. If $1 \leqq p, q \leqq \infty$ with $p \neq \infty$ and $q \neq 1$, these are equivalent:

(i) $\sup \left\{\| T_{W} t H_{q}: t \in T_{\Delta}(G)\right.$ and $\left.\|t\|_{p} \leqq 1\right\}<\infty$, 
(ii) $f \in L_{\Delta}^{p}(G)$ implies $T_{W} f \in L_{\Delta}^{q}(G)$,

(iii) $W \in\left(L_{\Delta}^{p}(G), L_{\Delta}^{q}(G)\right)$, and

(iv) $\left.W L^{q^{\prime}}(G)^{\wedge}\right|_{\Delta} \subseteq L^{p^{\prime}}(G)^{\wedge} \mid \Delta$.

Proof. (i) $\Rightarrow$ (ii). Let $\left\{t_{\alpha}\right\} \subseteq T(G)$ be an approximate identity (see [6], (28.53)). If $f \in L_{\Delta}^{p}(G)$ then $t_{\alpha} * f \in T_{\Delta}(G)$ hence by (i), for some $\kappa>0$

$$
\left\|T_{W}\left(t_{\alpha} * f\right)\right\|_{q} \leqq \kappa\left\|t_{\alpha} * f\right\|_{p} \leqq \kappa\|f\|_{p}
$$

By the weak compactness of norm balls in $L^{q}(G)(q \neq 1)$ there exists $g \in L^{q}(G)$ with $\|g\|_{q} \leqq \kappa\|f\|_{p}$ and $\hat{g}=W \hat{f}$. So by the uniqueness theorem, $T_{W} f=g \in L_{\Delta}^{q}(G)$.

(ii) $\Rightarrow$ (iii) is clear.

(iii) $\Rightarrow$ (iv). By hypothesis and the boundedness result 1.11, $T_{W}: L_{\Delta}^{p}(G) \rightarrow L_{\Delta}^{q}(G)$ is bounded and linear. So by 1.8 and 1.9 there is a bounded linear map $K: L^{q^{\prime}}(G)^{\wedge}\left|\Delta \rightarrow L^{p^{\prime}}(G)^{\wedge}\right| \Delta$ for which, whenever $f \in L^{q^{\prime}}(G)$ and $\chi \in \Delta, K(\hat{f} \mid \Delta)(\chi)=W(\chi) \hat{f}(\chi)$.

(iv) $\Rightarrow$ (i) follows similarly.

4.4. It is usually hard to identify $\left(E_{\Delta}, F_{\Delta}\right)$ even when $(E, F)$ is known (for $E, F \subseteq P M(G)$ ) so we pause to combine the approach of 3.1 with the result above.

Corollary. Let $1 \leqq p, q \leqq \infty$ with $p \neq \infty$ and $p \neq 1$. Then $W \in$ $\left(L_{\Delta}^{p}(G), L_{\Delta}^{q}(G)\right)$ iff $\sup \left\{\inf \left\{\|g\|_{p^{\prime}}: g \in L^{p^{\prime}}(G)\right.\right.$ and $\left.\hat{g}|\Delta=W \hat{f}| \Delta\right\}: f \in L^{q^{\prime}}(G)$ and $\left.\|f\|_{q^{\prime}} \leqq 1\right\} \equiv \sup \left\{\left\|T_{W} t\right\|_{q}: t \in T_{\Delta}(G)\right.$ with $\left.\|t\|_{p} \leqq 1\right\}<\infty$. $\left(L_{\Delta}^{p}(G), L_{\Delta}^{q}(G)\right)$ is a Banach space and when $p \leqq q$ it is a commutative Banach algebra which has an identity iff $\Delta \in \Lambda(q)$.

REMARKS. (i). Although $\left(L_{\Delta}^{p}(G), L_{\Delta}^{q}(G)\right)$ is unknown in general, special cases yield: $W \in\left(L_{\Delta}^{2}(G), L_{\Delta}^{2}(G)\right)$ iff $W \in l^{\infty}(\Delta)$; and for $1 \leqq p<\infty$, $W \in\left(L_{\Delta}^{p}(G), L_{\Delta}^{\infty}(G)\right)$ iff $W \in L^{p^{\prime}}(G)^{\wedge} \mid \Delta$ by [2], 16.7.5.

(ii). Conditions sufficient to ensure membership to $\left(L^{p}(\mathfrak{I}), L^{q}(\mathfrak{T})\right)$ are known and yield:

$$
\begin{aligned}
& \text { if } 1<p \leqq 2<q<\infty \text { and } W \in \mathbb{S}^{\lrcorner} \text {with } \\
& \quad \sup \left\{|W(n)|(1+|n|)^{1 / p-1 / q}: n \in \Delta\right\}<\infty
\end{aligned}
$$

then $W \in\left(L_{\Delta}^{p}(\mathfrak{I}), L_{\Delta}^{q}(\mathfrak{T})\right)$ - see [2], 16.4.6(3). More involved conditions apply when $q=p$.

4.5. When $p=1,4.3$ can be extended 'at each end'. 
CoROLlary. For $1<q<\infty$ these are equivalent:

(i ) $W \in\left(L_{\Delta}^{1}(G), L_{\Delta}^{q}(G)\right)$,

(ii ) $W M_{\Delta}(G)^{\wedge}\left|\Delta \leqq L_{\Delta}^{q}(G)^{\wedge}\right| \Delta$,

(iii) $W L^{q^{\prime}}(G)^{\wedge}\left|\Delta \leqq L^{\infty}(G)^{\wedge}\right| \Delta$,

(iv) $W L^{q^{\prime}}(G)^{\wedge}\left|\Delta \leqq C(G)^{\wedge}\right| \Delta$.

Proof. (i) $\Rightarrow$ (ii) follows as in $4.3(\mathrm{i}) \Longrightarrow$ (ii).

(ii) $\Rightarrow$ (iii). Since (ii) $\Rightarrow$ (i), 4.3 implies this.

(iii) $\Rightarrow$ (iv). If $f \in L^{q^{\prime}}(G)$, by [6], $(32 \cdot 30)$, there exist $g \in L^{1}(G)$ and $f_{0} \in L^{q^{\prime}}(G)$ with $f=g * f_{0}$. By (iii) there is $h_{0} \in L^{\infty}(G)$ with $W \hat{f}_{0}\left|\Delta=\hat{h}_{0}\right| \Delta$. Setting $h=g * h_{0}$ gives $h \in C(G)$ and

$$
\hat{h}\left|\Delta=\hat{g} \hat{h}_{0}\right| \Delta=\hat{g} W \hat{f}_{0}|\Delta=W \hat{f}| \Delta
$$

as required.

4.6. More can also be said when $p=2$.

THEOREM. For $1<q \leqq \infty, W \in\left(L_{A}^{2}(G), L_{\Delta}^{q}(G)\right)$ iff for all $f \in L^{q^{\prime}}(G)$,

$$
\left(\sum_{\chi \in \Delta}|W(\chi) \hat{f}(\chi)|^{2}\right)^{1 / 2} \leqq \kappa\|f\|_{q^{\prime}}
$$

for some constant $\kappa$.

Proof. $(\Longrightarrow)$ uses the adjoint of $T_{W}$ as in 4.3 (iii) $\Rightarrow$ (iv).

$(\Longleftrightarrow)$. Parseval's identity with the hypothesis shows $W L^{q^{\prime}}(G)^{\wedge} \mid \Delta \leqq$ $L^{2}(G)^{\wedge} \mid \Delta$ hence by $4.3(\mathrm{iv}), W \in\left(L_{\Delta}^{2}(G), L_{\Delta}^{q}(G)\right)$.

Note. By choosing an approximate identity the method above shows $W \in\left(L_{\Delta}^{2}(G), L_{\Delta}^{\infty}(G)\right)$ iff $W \in l^{2}(\Delta)$, as noted in $4.4(\mathrm{i})$.

Since $\left.\left(L_{\Delta}^{1}(G)\right), L_{\Delta}^{\infty}(G)\right) \subseteq\left(L_{\Delta}^{2}(G), L_{\Delta}^{\infty}(G)\right)$ we have thus dealt with the case $q=\infty$ of 4.5. Alternatively,

$$
\left(L_{\Delta}^{p}(G), L_{\Delta}^{\infty}(G)\right) \leqq l^{2}(\Delta) \text { when } 1 \leqq p \leqq 2 .
$$

See also 4.8 .

4.7. Summarising what we have gleaned about $W$-Sidon sets by virtue of 4.1:

CoROLlary. If $\Delta$ is W-Sidon then

(i ) for all $\mu \in M_{\Delta}(G), T_{W} \mu \in L_{\Delta}^{2}(G)$ and $\left\|T_{W} \mu\right\|_{2} \leqq 8\|W\|\left\|_{\Delta}\right\| \mu \|$,

(ii) for all $f \in L_{\Delta}^{2}(G), T_{W} f \in L_{\Delta}^{p}(G)$ whenever $2<p<\infty$ and $\left\|T_{W} f\right\|_{p} \leqq 2\|W\|_{\Delta} p^{1 / 2}\|f\|_{2}$,

(iii) for all $\mu \in M_{\Delta}(G), T_{W^{2}} \mu \in L_{\Delta}^{p}(G)$ whenever $2<p<\infty$ and $\left\|T_{W^{2}} \mu\right\|_{p} \leqq 16\|W\|_{\Delta}^{2} p^{1 / 2}\|\mu\|_{\text {, }}$ 
(iv) for all $\phi \in l^{2}(\Delta)$, there is $f \in C(G)$ such that $\hat{f} \mid \Delta=W \dot{\phi}$ and $\|f\|_{\infty} \leqq 8\|W\|_{\Delta}\|\phi\|_{2}$, and

( v) if $1<p \leqq 2$ and $f \in L^{p}(G)$ then

$$
\left(\sum_{\chi \in \Delta}|W(\chi) \hat{f}(\chi)|^{2}\right)^{1 / 2} \leqq 2\|W\|_{\Delta} p^{-1 / 2}\|f\|_{p} .
$$

Proof. All are obvious except possibly (iii). If $\mu \in M_{\Delta}(G)$ and $2<p<\infty$, by (i) and (ii),

$$
\begin{aligned}
\left\|T_{W^{2}} \mu\right\|_{p} & =\left\|T_{W}\left(T_{W} \mu\right)\right\|_{p} \leqq 2\|W\|_{\Delta} p^{1 / 2}\left\|T_{W} \mu\right\|_{2} \\
& \leqq 16\|W\|_{\Delta}^{2} p^{1 / 2}\|\mu\| .
\end{aligned}
$$

4.8. For which $W$ can 1.10 (vi) be tightened to

$$
W L_{\Delta}^{p}(G)^{\wedge} \mid \Delta \subseteq l^{1}(\Delta)
$$

for some $p \in[1, \infty)$ ? We show that when $1 \leqq p \leqq 2$, (4.8.1) holds iff $\Delta$ is a trivial $W$-Sidon set, and we give a partial answer when $2<p<\infty$.

THEOREM. If $\Delta \subseteq X$ then

(i ) $1 \leqq p<\infty$ implies $\left(L_{\lrcorner}^{p}(G), A_{\Delta}(G)\right) \leqq L^{p \prime}(G)^{\wedge} \mid \Delta$,

(ii) $1 \leqq p \leqq 2$ implies $l^{p}(\Delta) \leqq\left(L_{4}^{p}(G), A_{\Delta}(G)\right)$,

(iii) $2<p<\infty$ implies $l^{2}(\Delta) \cong\left(L_{\Delta}^{p}(G), A_{\Delta}(G)\right)$, and

(iv) $2<p<\infty$ implies $\left(L_{\Delta}^{p}(G), A_{\Delta}(G)\right) \cap\left(L_{\Delta}^{2}(G), L_{\Delta}^{p^{\prime}}(G)\right) \subseteq l^{4}(\Delta)$.

Proof. (i) This follows by 4.4(i) but may be proved quickly as follows. If $W \in\left(L_{\Delta}^{p}(G), A_{\Delta}(G)\right)$ then letting $K$ denote the composition of the isomorphism of 1.8 (i) with $T_{W}^{*}$, we have $K: l^{\infty}(\Delta) \rightarrow L^{p^{\prime}}(G)^{\wedge} \mid \Delta$ and whenever $\phi \in l^{\infty}(\Delta)$ and $\chi \in \Delta,(K \phi)(\chi)=W(\chi) \phi(\chi)$. Taking $\dot{\phi}=\mathbf{1}$ this gives

$$
f \in L^{p^{\prime}}(G) \text { with } \hat{f} \mid \Delta=W
$$

as required.

(ii) If $1 \leqq p \leqq 2$ and $f \in L_{\lrcorner}^{p}(G)$ then by the Hausdorff-Young theorem and Hölder's inequality, whenever $W \in l^{p}(\Delta)$,

$$
\sum_{\chi \in \Delta}|W(\chi) \hat{f}(\chi)| \leqq\|W\|_{p}\|f\|_{p}<\infty .
$$

(iii) If $2<p<\infty$ and $f \in L_{\lrcorner}^{p}(G)$ then $\hat{f} \mid \Delta \in l^{2}(\Delta)$ hence when $W \in l^{2}(\Delta)$,

$$
\sum_{\chi \in \Delta}|W(\chi) \hat{f}(\chi)| \leqq\|W\|_{2}\|\hat{f}\|_{2}<\infty .
$$

(iv) Continuing from (4.8.2), if $2<p<\infty$, 4.6 shows 


$$
\left(\sum_{\chi \in\lrcorner}|W(\chi)|^{4}\right)^{1 / 2} \leqq 2\|W\|_{\Delta} p^{1 / 2}\|f\|_{p^{\prime}},
$$

so $W \in l^{4}(\Delta)$.

Remarks. (i) Taking $W$ constant, (4.8.2) shows there can be no infinite Sidon sets $\Delta$ with $L_{4}^{p}(G)^{\wedge} \mid \Delta \leqq l^{1}(\Delta)$ when $1 \leqq p<\infty$.

(ii) Results (i) and (ii) above combine to show that trivial $W$-Sidon sets are precisely the $W$-Sidon sets for which (4.8.1) holds when $p \in[1,2]$.

Results (iii) and (iv) do not interlock in this way but show, thanks to $4.7(\mathrm{v})$, that when $p \in(2, \infty),(4.8 .1)$ cannot hold when $\Delta$ is $W$-Sidon and $W \notin l^{4}(\Delta)$.

(iii) For comparison, $\left(L_{\Delta}^{p}(G), A_{\Delta}(G)\right)$ is identified when $\Delta$ is a subgroup of $X$ in [6], $(36 \cdot 20)$ via the method of 1.3 .

4.9. When $W=1$ the inclusions implied by 4.7 for Sidon sets are, by Parseval's identity, equalities. In fact these are the only $W$-Sidon sets with equality:

THEOREM. $\Delta$ is Sidon whenever it is W-Sidon and one of these holds.

( i ) $l^{2}(\Delta) \subseteq W M_{\Delta}(G)^{\wedge} \mid \Delta$,

(ii) $L^{\infty}(G)^{\wedge} \mid \Delta \subseteq W l^{2}(\Delta)$,

(iii) $C(G)^{\wedge} \mid \Delta \subseteq W l^{2}(\Delta)$,

(iv) $L_{\Delta}^{p}(G)^{\wedge} \mid \Delta \leqq W l^{2}(\Delta)$, for some $p \in(2, \infty)$ and

( v ) $\quad l^{2}(\Delta) \subseteq W L^{p}(G)^{\wedge} \mid \Delta$, for some $p \in(1,2)$.

Proof. Theorem 1.12 as used in 1.14 makes short work of these.

4.10. So far we have discussed the behaviour of $T_{W} \pi$ when $\pi$ is a $\Delta$-spectral measure of $L^{p}$-function and $\Delta$ is $W$-Sidon. Immediately from 1.10 (viii) we have: $\Delta$ is $W$-Sidon iff $W P M_{\Delta}(G)^{\wedge}\left|\Delta \subseteq M(G)^{\wedge}\right| \Delta$. From 1.14(i) this inclusion is proper whenever $\Delta$ is not Sidon.

Evidently $T_{W}\left(P M_{\Delta}(G)\right) \subseteq L_{\Delta}^{2}(G)$ iff $\Delta$ is a trivial $W$-Sidon set and if $T_{W}\left(P M_{\Delta}(G)\right) \cong M_{\Delta}(G)$ then $W \in l^{4}(\Delta)$.

4.11. We now deduce more about those $W$ in $\mathfrak{M}(\Delta)$. Specialising to $\mathfrak{I}$ (though (4.11.1) holds in general) we use:

THEOREM. Let $F \in \mathbb{C}^{\beta}$. If $\phi F \in \bigcap\left\{L^{p}(\mathfrak{I})^{\wedge}: 1 \leqq p<\infty\right\}$ for all $\phi \in c_{0}(3)$ then for all $\alpha>0, \sum_{n \neq 0}\left|n^{-\alpha} F(n)\right|<\infty$.

Proof. Successive applications of 1.11 and 1.8 show that if 
$1<p<\infty$, then $\phi F \in L^{p}(\mathfrak{T})^{\wedge}$ for all $\phi \in c_{0}(3)$ implies $W L_{\Delta}^{p^{\prime}}(G)^{\wedge} \mid \Delta \subseteq l^{1}(\Delta)$. So the hypothesis entails

(4.11.1) for all $p \in(1, \infty)$ and all $g \in L^{p}(\mathfrak{T}), \sum_{n \in \mathbb{B}}|F(n) \hat{g}(n)|<\infty$.

Now if $0<\alpha<1$ then by [2], Exercise 7.8, there exist $p \in\left(1,(1-\alpha)^{-1}\right)$ and $g \in L^{p}(\mathfrak{T})$ such that $\hat{g}(n)=n^{-\alpha}$ for $n \neq 0$. If $\alpha \geqq 1$ then the map $n \mapsto n^{-\alpha}$ belongs to $l^{2}(3 \backslash\{0\})$ hence there is $g \in L^{2}(\mathfrak{I})$ with $\hat{g}(n)=n^{-\alpha}$ whenever $n \neq 0$.

In either case, substitution into (4.11.1) yields

$$
\sum_{n \neq 0}\left|F(n) n^{-\alpha}\right|<\infty
$$

as required.

Notes. (i) In [12] we show the converse of this theorem to be false.

(ii) The sum $\sum_{n \neq 0}\left|n^{-\alpha} F(n)\right|$ was first considered by Hardy and Littlewood in [5]. Their results imply that it is finite whenever $\alpha>1 / 2$ and may be infinite otherwise, when $F \in \bigcap\left\{L^{p}(\mathfrak{I})^{\wedge}: 1 \leqq p<\infty\right\}$.

4.12. The information this gives about $W$ is:

CoRollary. If $W \in \mathfrak{W}(\Delta)$ then for all $\mu \in M_{\Delta}(\mathfrak{T})$, if $\alpha>0$ then

$$
\sum_{n \neq 0}\left|n^{-\alpha} \hat{\mu}(n) W^{2}(n)\right|<\infty \text {. }
$$

Proof. In fact if $\phi \in l^{\infty}(3)$ (not merely $c_{0}(3)$ ) and $\Delta$ is $W$-Sidon

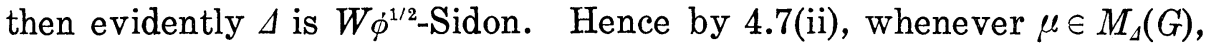

$$
\phi W^{2} \hat{\mu} \in \bigcap\left\{L_{\Delta}^{p}(G)^{\wedge}: 1 \leqq p<\infty\right\}
$$

so the conclusion follows from 4.11.

4.13. Using $l^{\infty}(3)$ rather than $c_{0}(3)$ above seems to be stronger. However in this context they are equivalent.

Theorem. Let $F \in \mathbb{C}^{x}$. Then $\phi F$ belongs to $\bigcap\left\{L^{p}(G)^{\wedge}: 1 \leqq p<\infty\right\}$ for all $\phi \in c_{0}(X)$ iff it does for all $\phi \in l^{\infty}(X)$.

Proof. This follows readily upon taking the bidual of the map $K: c_{0}(X) \rightarrow L^{p}(G)$ given by $(K \phi)^{\wedge}=\phi F$.

4.14. It might be hoped that a tight necessary condition for $W$ to belong to $\mathfrak{W}(\Delta)$ follows from 4.12 by eliminating $\mu$ somehow to give a purely combinatorial property. However the $\Delta$-spectral 
measures compensate for variations in the thickness of $\Delta$, so we turn to other means for this.

Refer to [3], 3.1 for the definition of a test family of order $m$.

THEOREM. If $W \in\left(L_{\Delta}^{p}(G), L_{\Delta}^{q}(G)\right)$ where $1 \leqq p \leqq 2$ and $1<q<\infty$, and $\mathfrak{F}$ is a test family of order $m$ then for each $\Phi \in \mathfrak{F}$,

$$
\sum_{\chi \in \Phi \cap \Delta}|W(\chi)|^{2} \leqq \kappa^{2} m \nu(\Phi)^{2 / q}
$$

where $\kappa$ is the unnamed constant in 4.4.

Proof. This is a routine modification of [3], 3.2 for which details appear in [11].

COROLLARY 4.15. If $\Delta$ is $W$-Sidon and $\mathfrak{F}$ is a test family of order $m$ then for each $\Phi \in \mathfrak{F}$ with $\nu(\Phi) \geqq 3$,

$$
\sum_{\chi \in \Phi \cap \Delta}|W(\chi)|^{2} \leqq 8 e\|W\|_{\Delta} m \log \nu(\Phi) \text {. }
$$

Proof. By hypothesis and 4.7(ii), $W \in\left(L_{\Delta}^{2}(G), L_{\Delta}^{q}(G)\right)$ whenever $q \in(2, \infty)$ and so by 4.14 ,

$$
\sum_{\chi \in \Phi_{\cap} \Delta}|W(\chi)|^{2} \leqq 4\|W\|_{\Delta}^{2} q m \nu(\Phi)^{2 / q}
$$

Taking $q=2 \log \nu(\Phi)$ so that $q>2$ because $\nu(\Phi) \geqq 3$, this entails the result.

Notes. (i). This means that if $\varepsilon>0$, the number of elements of $\Delta$ in $\Phi$ with $|W(\chi)|>\varepsilon$ remains small as $\Phi$ enlarges.

(ii). For $q=\infty$ the result above is overshadowed by the note to 4.6 .

The results of this paper appear in [11]. The author is deeply indebted to his supervisor, Professor R. E. Edwards, for his suggestions and encouragement. $\mathrm{He}$ is also grateful to Dr. J. R. McMullen for a correction to 1.7 and to the referee for several improvements.

\section{REFERENCES}

1. R. E. Edwards, Changing signs of Fourier coefficients, Pacific J. Math., 15 (1965), 463-475.

2. - Fourier Series. A Modern Introduction, I and II, Holt, Rinehart and Winston Inc., New York, 1967.

3. — E. Hewitt and K. A. Ross, Lacunarity for compact groups I, Indiana Univ. Math. J., 21 (1972), 787-806.

4. R. E. Edwards and K. A. Ross, p-Sidon sets, J. Functional Analysis, 15 (1974), 404-427. 
5. G. H. Hardy and J. E. Littlewood, Some new properties of Fourier constants, Math. Ann., 97 (1927), 159-209.

6. E. Hewitt and K. A. Ross, Abstract Harmonic Analysis, Springer-Verlag, Berlin, 1963 and 1970.

7. Y. Katznelson, An Introduction to Harmonic Analysis, John Wiley and Sons Inc., New York, 1968.

8. G. Köthe, Topological Vector Spaces I, Springer-Verlag, Berlin, 1969.

9. W. Rudin, Trigonometric series with gaps, J. Math. Mech., 9 (1960), 203-227.

10. - Fourier Analysis on Groups, Interscience, New York, 1962.

11. J. W. Sanders, Some lacunary and random Fourier series, Ph. D. Dissertation, Australian National University, January, 1975.

12. - Unbounded operators and random Fourier series, Math. Proc. Cambridge Philos. Soc., to appear.

13. N. Th. Varopoulos, Sidon sets in $R^{n}$, Math. Scand., 27 (1970), 39-49.

Received May 6, 1975.

The Australian National University 



\section{PACIFIC JOURNAL OF MATHEMATICS}

EDITORS

RICHARD ARENS (Managing Editor)

University of California

Los Angeles, California 90024

R. A. BEAUMONT

University of Washington

Seattle, Washington 98105
J. DugundJI

Department of Mathematics

University of Southern California

Los Angeles, California 90007

D. Gilbarg and J. Milgram

Stanford University

Stanford, California 94305

\section{ASSOCIATE EDITORS}

E. F. BECKENBACH

B. H. NeumanN

F. WOLF

K. YosHIDA

\section{SUPPORTING INSTITUTIONS}

UNIVERSITY OF BRITISH COLUMBIA

UNIVERSITY OF SOUTHERN CALIFORNIA

CALIFORNIA INSTITUTE OF TECHNOLOGY

UNIVERSITY OF CALIFORNIA

STANFORD UNIVERSITY

UNIVERSITY OF TOKYO

MONTANA STATE UNIVERSITY

UNIVERSITY OF UTAH

UNIVERSITY OF NEVADA

WASHINGTON STATE UNIVERSITY

NEW MEXICO STATE UNIVERSITY

UNIVERSITY OF WASHINGTON

OREGON STATE UNIVERSITY

UNIVERSITY OF OREGON

OSAKA UNIVERSITY

AMERICAN MATHEMATICAL SOCIETY
NAVAL WEAPONS CENTER

Printed in Japan by International Academic Printing Co., Ltd., Tokyo, Japan 


\section{Pacific Journal of Mathematics}

\section{Vol. 63, No. $1 \quad$ March, 1976}

Ralph Artino, Gevrey classes and hypoelliptic boundary value problems ....... 1

B. Aupetit, Caractérisation spectrale des algèbres de Banach commutatives .... 23

Leon Bernstein, Fundamental units and cycles in the period of real quadratic

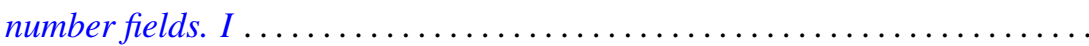

Leon Bernstein, Fundamental units and cycles in the period of real quadratic number fields. II.................................... 63

Robert F. Brown, Fixed points of automorphisms of compact Lie groups ........

Thomas Ashland Chapman, Concordances of noncompact Hilbert cube

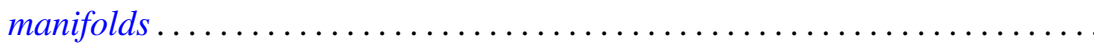

William C. Connett, V and Alan Schwartz, Weak type multipliers for Hankel

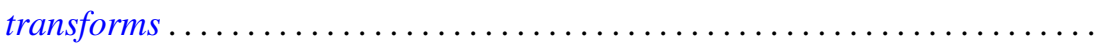

John Wayne Davenport, Multipliers on a Banach algebra with a bounded approximate identity .....................................

Gustave Adam Efroymson, Substitution in Nash functions ................ 137

John Sollion Hsia, Representations by spinor genera ..................

William George Kitto and Daniel Eliot Wulbert, Korovkin approximations in

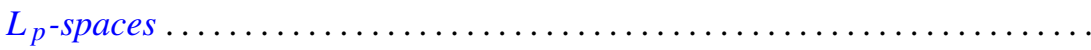

Eric P. Kronstadt, Interpolating sequences for functions satisfying a Lipschitz. condition ...........................................

Gary Douglas Jones and Samuel Murray Rankin, III, Oscillation properties of certain self-adjoint differential equations of the fourth order...

Takaŝi Kusano and Hiroshi Onose, Nonoscillation theorems for differential

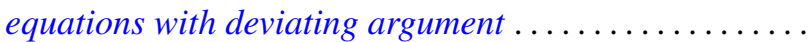

David C. Lantz, Preservation of local properties and chain conditions in commutative group rings. ...

Charles W. Neville, Banach spaces with a restricted Hahn-Banach extension property....

Norman Oler, Spaces of discrete subsets of a locally compact group ...

Robert Olin, Functional relationships between a subnormal operator and its minimal normal extension.

Thomas Thornton Read, Bounds and quantitative comparison theorems for nonoscillatory second order differential equations ...... .

Robert Horace Redfield, Archimedean and basic elements in completely distributive lattice-ordered groups...

Jeffery William Sanders, Weighted Sidon sets

Aaron R. Todd, Continuous linear images of pseudo-complete linear topological spaces.

J. Jerry Uhl, Jr., Norm attaining operators on $L^{1}[0,1]$ and the Radon-Nikodým property.

William Jennings Wickless, Abelian groups in which every endomorphism is a left multiplication. 